\title{
Logistical Simulation Modeling for Planning a Soil Remediation Process
}

\author{
David Kessel, Jihan Jeon, Jaeyeon Jung, Eutteum Oh, and Chang-Lak Kim \\ KEPCO International Nuclear Graduate School, 658-91 Haemaji-ro, Seosaeng-myeon, Ulju-gun, Ulsan 54014, Republic of Korea
}

Correspondence should be addressed to Chang-Lak Kim; clkim@kings.ac.kr

Received 15 November 2018; Revised 11 March 2019; Accepted 9 April 2019; Published 2 May 2019

Guest Editor: Rema Abdulaziz

Copyright (C) 2019 David Kessel et al. This is an open access article distributed under the Creative Commons Attribution License, which permits unrestricted use, distribution, and reproduction in any medium, provided the original work is properly cited.

This paper describes the development of a discrete event simulation model using the FlexSim software to support planning for soil remediation at Korean nuclear power plants that are undergoing decommissioning. Soil remediation may be required if site characterization shows that there has been radioactive contamination of soil from plant operations or the decommissioning process. The simulation model was developed using a dry soil separation and soil washing process. Preliminary soil data from the Kori 1 nuclear power plant was used in the model. It was shown that a batch process such as soil washing can be effectively modeled as a discrete event process. Efficient allocation of resources and efficient waste management including volume and classification reduction can be achieved by use of the model for planning the soil remediation process. Cost will be an important criterion in the choice of suitable technologies for soil remediation but is not included in this conceptual model.

\section{Introduction}

This paper describes the development of a conceptual simulation model that can be used for logistics planning for site remediation at Korean nuclear power plants and specifically the Kori 1 reactor site. A FlexSim discrete event simulation model of the site soil remediation and waste management process was developed to support optimization of performance, resources, and time and to minimize nuclear waste. Cost will be an important criterion in the choice of suitable technologies for soil remediation but is not included in this first conceptual model.

Kori Unit 1, the oldest nuclear power plant (NPP) in Korea, was permanently shut down in June 2017. The decommissioning of Kori Unit 1 will follow in the future and site remediation will be carried out at the end of the decommissioning project.

Operation of a nuclear power plant may lead the release of radioactive materials to the soil and groundwater at the site which could migrate to the surrounding environment. Small radioactive releases may not be discovered until the NPP undergoes decommissioning and must be remediated to prevent migration of radioactive materials [1].

Nuclear power plant site remediation is performed after site structures have been removed and is the last step in decommissioning. Remediation may be required remove contamination from the site soil to allow the reuse of the site subject to the regulatory requirements for site release.

Site remediation has two safety-related objectives. One is to reduce radiation exposure to workers and the general public, and the other is to reduce the radioactivity to the level required for the intended use of the site after its release.

Korea does not have domestic experience in NPP decommissioning and site remediation. Furthermore, the decommissioning and remediation work at Kori 1 has not started and so the site soil and groundwater conditions have not been characterized. The extent and nature of radioactive contamination at the site is uncertain, and as such the development of this simulation model must be preliminary. This does not diminish the fact that effective planning is necessary to reduce project uncertainty and risk to achieve the site remediation objectives.

A review of the literature for site remediation has shown that simulation modeling is a useful tool for planning decommissioning and site remediation. The literature has also shown the value of simulation models for design and optimization of industrial processes. In order to develop a preliminary simulation model, soil washing was chosen for the remediation technology. 


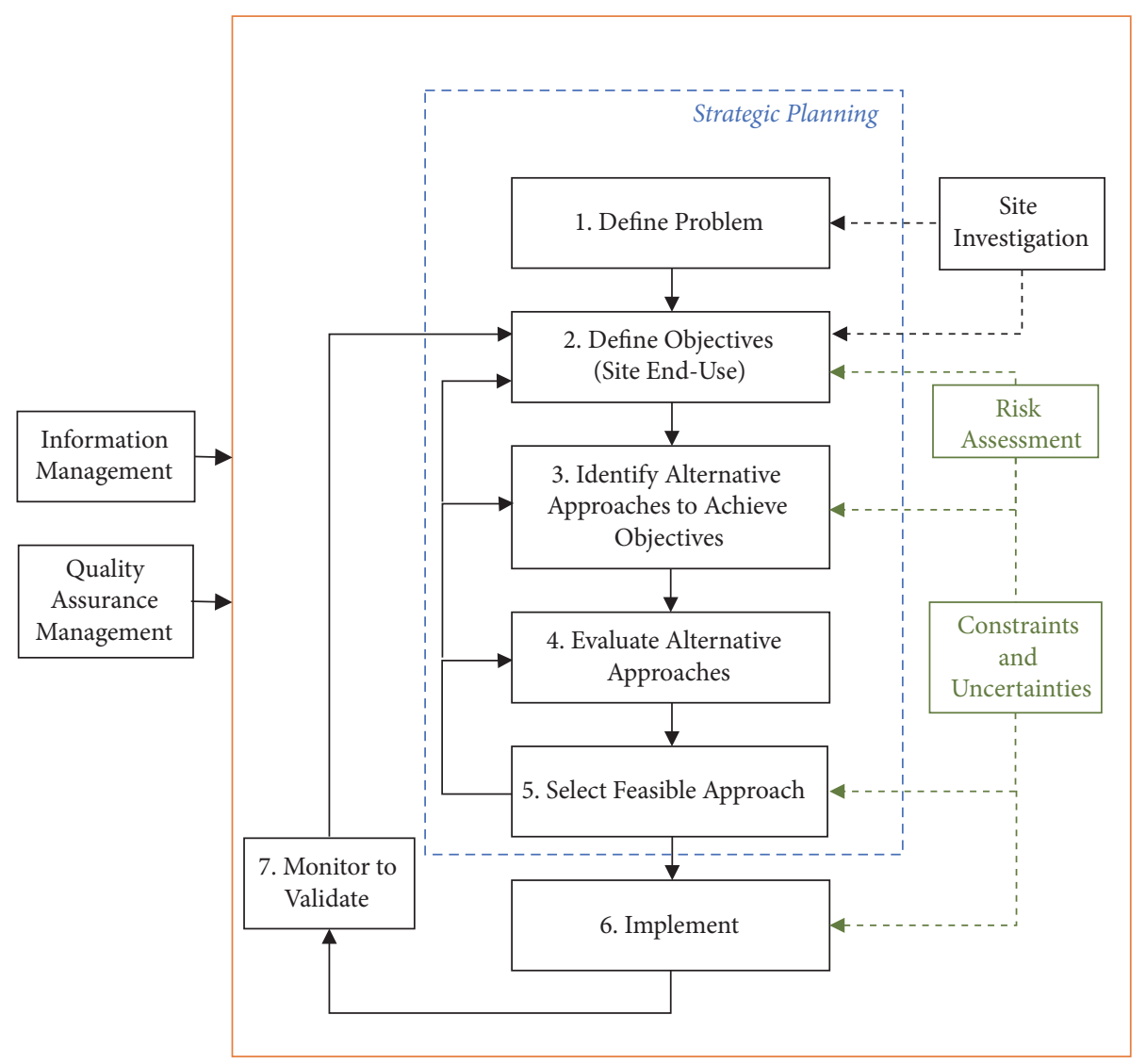

FIGURE 1: IAEA integrated planning process [1].

Once decommissioning commences at the Kori 1 site, the simulation model can be further developed to include actual site conditions and data for radionuclide contamination.

This paper includes a literature review of remediation planning, soil remediation technology, and the application of discrete event simulation for nuclear-related industrial process operations. Selection of the appropriate technology and a conceptual process model based on soil washing is presented. A discrete event simulation model was developed and verified.

\section{Literature Review}

International and Korean domestic experience in remediation planning, applicable technologies, and the use of discrete event simulation for remediation projects was reviewed.

2.1. Planning for Remediation. The International Atomic Energy Agency (IAEA) defined soil remediation as an iterative process that includes identification of contaminants, spatial distribution, appropriate decontamination technology, performing remediation, verification of effectiveness, and postremediation monitoring. IAEA recommended that plans for decommissioning and remediation should consider the interfaces and interactions between both processes to improve effectiveness and efficiency and reduce overall costs. The IAEA process for site remediation is shown in Figure 1.
The figure depicts an iterative decision process for selecting the appropriate technology. IAEA recommended that decision tools such as simulation modeling, multicriteria decision analysis, and risk assessment be used to select the remediation technology [1].

The OECD Nuclear Energy Agency (NEA) defines remediation as a six-phase process as shown in Figure 2 [3]. The phases are defined as follows:

(i) Phase 1: problem identification

(ii) Phase 2: remedial investigation (assessment and characterization)

(iii) Phase 3: remedy planning (alternative evaluation and selection)

(iv) Phase 4: remedial action (implementation)

(v) Phase 5: project closeout

(vi) Phase 6: institutional control

The Nuclear Energy Agency (NEA) recommended the development of a conceptual site model (CSM) as the as part of an overall site remediation process as shown in Figure 2. The CSM is based on characterization data including site geology, soil classification, and contaminant type, concentration, and spatial distribution. The CSM is used to guide remediation planning and implementation [3]. 


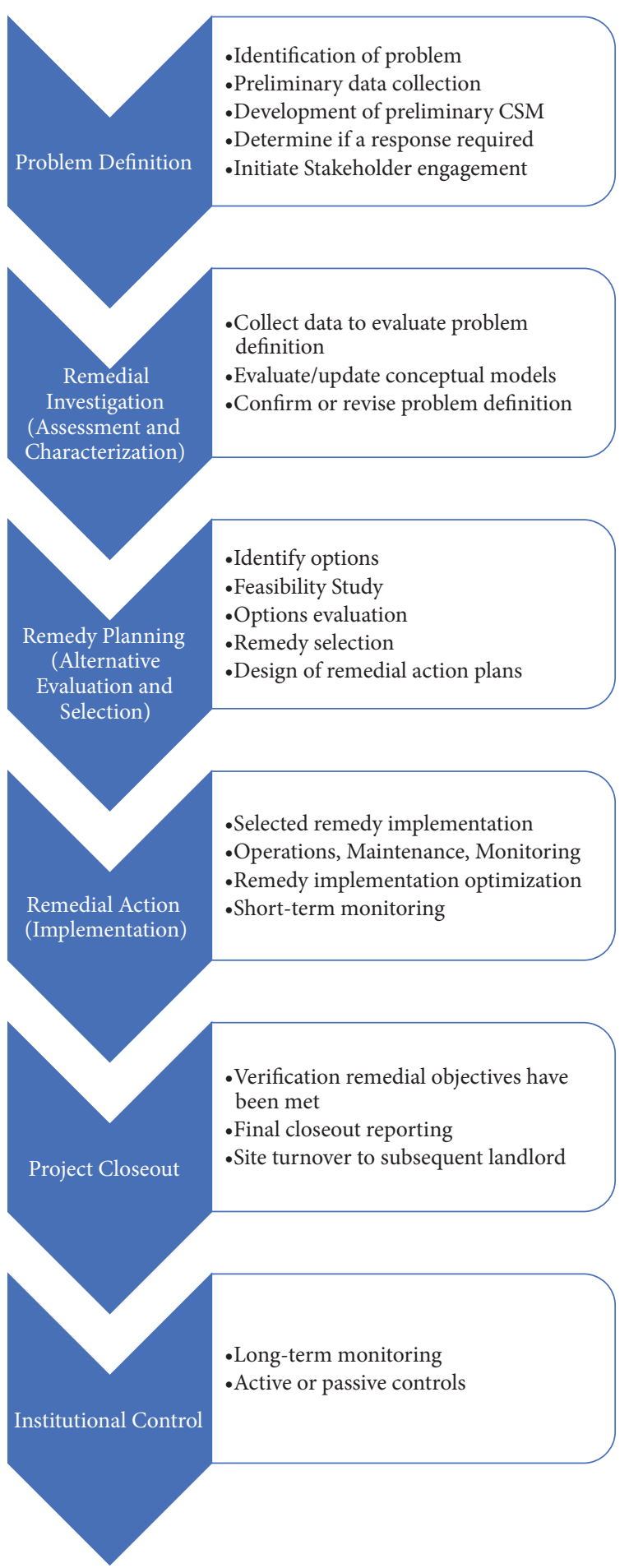

Figure 2: NEA nuclear site remediation phases [3].

The Electric Power Research Institute (EPRI) summarized the experience in US decommissioning and site remediation projects. EPRI found that contamination below structures was difficult to identify and characterize until after the structures are removed in the decommissioning phase. Therefore, site characterization must be an iterative process performed throughout a decommissioning project [4].
2.2. Soil Remediation Technologies. The US EPA issued the Technology Reference Guide for Radioactively Contaminated Media that summarized technologies for the remediation of radioactive contamination in soil and groundwater. The guide focused on existing technologies rather than experimental approaches [5].

The guide identified solvent extraction (Figure 3) as an established ex situ technology to remove radionuclides from soils to reduce the volume of nuclear waste or reduce its classification. Solvent extraction has been widely used in the nuclear industry, for example, in conventional uranium milling operations. Solvent extraction, when used for soil remediation, is operated as a batch process to remove the radionuclides. The solvent containing the contaminants is then volume reduced for disposal.

Other methods can be used in combination with solvent extraction. These include physical separation and water washing [5].

Physical separation is an established technology for removing radionuclides from soil. Radionuclides preferentially adsorb soil fines (silts and clays) as opposed to the course fraction (sand and gravel). Thus, the fine-grained soils tend to capture the radionuclides, and physically separating the fine and course fractions is the basis for decontamination. The simplest physical separation method is dry separation that uses sieving with successively finer screens to separate the fines from course fraction. This method concentrates the contaminated soil and reduces the volume of soil for treatment or disposal [5].

A more complex dry separation method, the segmented gate system, uses radiation detectors to improve the separation factor for some radionuclides (Figure 4). This method effectively treats soils contaminated with gamma emitting radionuclides. This method is normally only used for soil contaminated with no more than two radionuclides with different gamma energies [5].

Soil washing is an established technology that is often used in combination with dry separation. Soil washing is an ex situ process using water and surfactants to remove radionuclides from soil. Soil washing can reduce bulk soil or minimize the waste by selectively removing fine-grained particles (silts and clays), which contain most of the contamination, from the bulk soil. It is possible based on the principle that contaminants are generally bound more tightly to the fine-grained particles and not to the larger course grained.

The contaminated wash solution can be volume reduced for disposal. Soil washing is more effective for soils containing less than $25 \%$ fines and more than $50 \%$ course material. Effectiveness diminishes for soils with more than $10 \%$ total organics. Soil washing has been used to treat soil contaminated with radionuclides including plutonium, radium, uranium, thorium, technetium, strontium, and cesium [5]. A process flow diagram of the soil washing process is shown in Figure 5.

The electrokinetic remediation (ER) process has been used to remove radionuclides from low permeability soil. The ER process is an emerging in situ or ex situ soil remediation technology that can separate radionuclides from soil, sludge, and sediment (Figure 6). ER removes metals and organic 


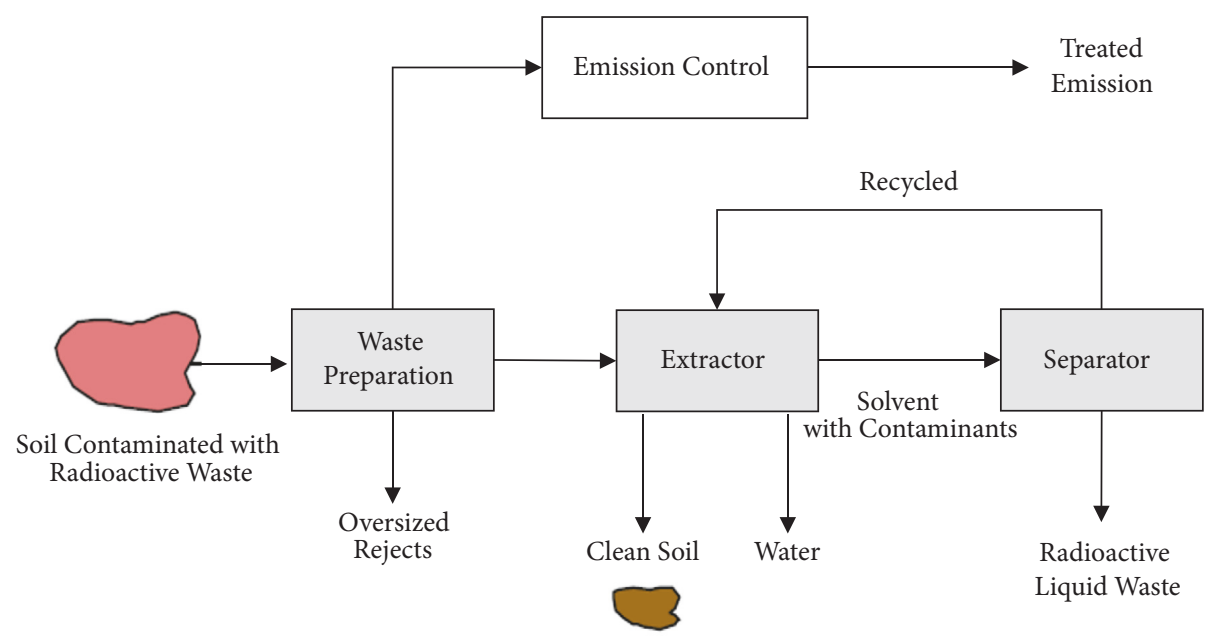

FIgURE 3: Solvent extraction.

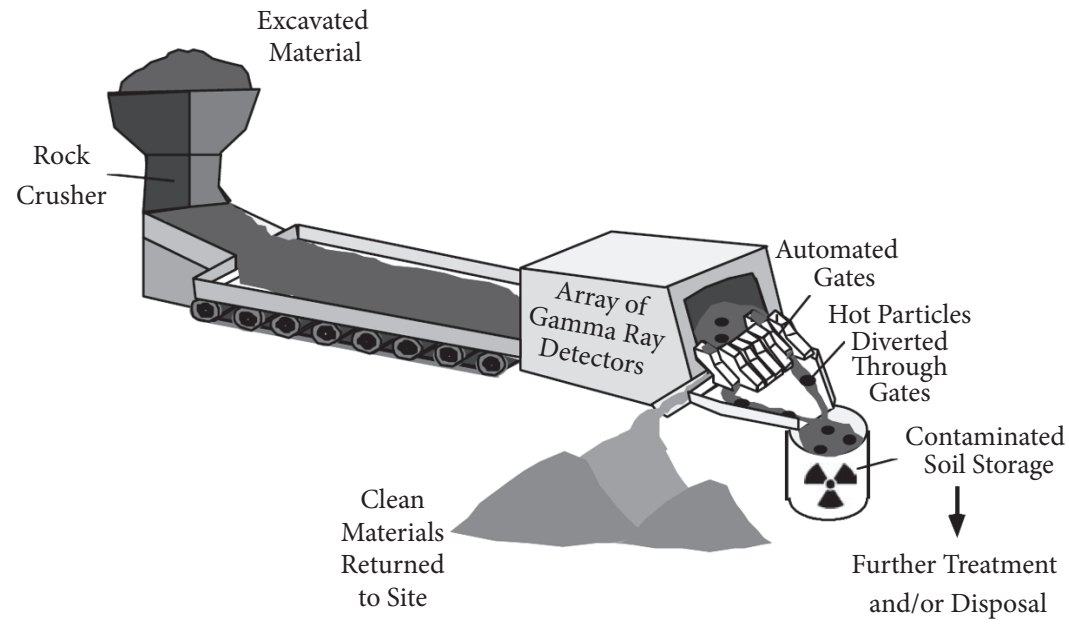

FIGURE 4: Segmented gate dry soil separation [5].

contaminants from low permeability soil. ER uses electrochemical and electrokinetic processes to desorb, and then remove, metals and polar organics. In situ ER is performed by applying low voltage direct current across electrode pairs that are implanted in the ground containing contaminated soil. The current carries ions and charged compounds to the electrodes. The negatively charged contaminants move to the cathode and the positively charged anions move to the anode.

The Korean Atomic Energy Research Institute (KAERI) has developed an ex situ electrochemical method using soil washing, electrokinetic separation, and waste solution treatment for soils contaminated with radionuclides. This method has been tested successfully at the bench scale [10].

Electrokinetic technology has been demonstrated at the pilot scale and at full scale at several US sites. Geokinetics International Inc. has demonstrated an in situ electrokinetic remediation process at five sites in Europe [11].

2.3. Discrete Event Simulation for Soil Remediation. Simulation models are an abstraction of the real system that represent the key characteristics and features of a specific system or process. Simulation models may be discrete or continuous.

Discrete event simulation (DES) uses a transaction-based approach to model the dynamic behavior of a system. In discrete event simulation state changes occur only at discrete times as opposed to continuous simulations in which the state variables change continuously. DES is an appropriate tool for modeling batch processing systems [12].

Heilala et al. discussed the use of DES as a system analysis tool to evaluate production system concepts, system configuration, and control logic. Simulation models provide the ability to evaluate the throughput of a system, identify bottlenecks, and answer "what-if" questions about proposed changes to the system. Process of optimization requires decisions to be made in a comprehensive way, including budget, schedules, and possible resources such as engineers and technologies. Figure 7 shows the potential for using simulation in the planning phase [7].

Dottavio et al. have demonstrated the application of DES for nuclear power plant decommissioning, site remediation, and nuclear waste management. Nuclear waste flows for a 


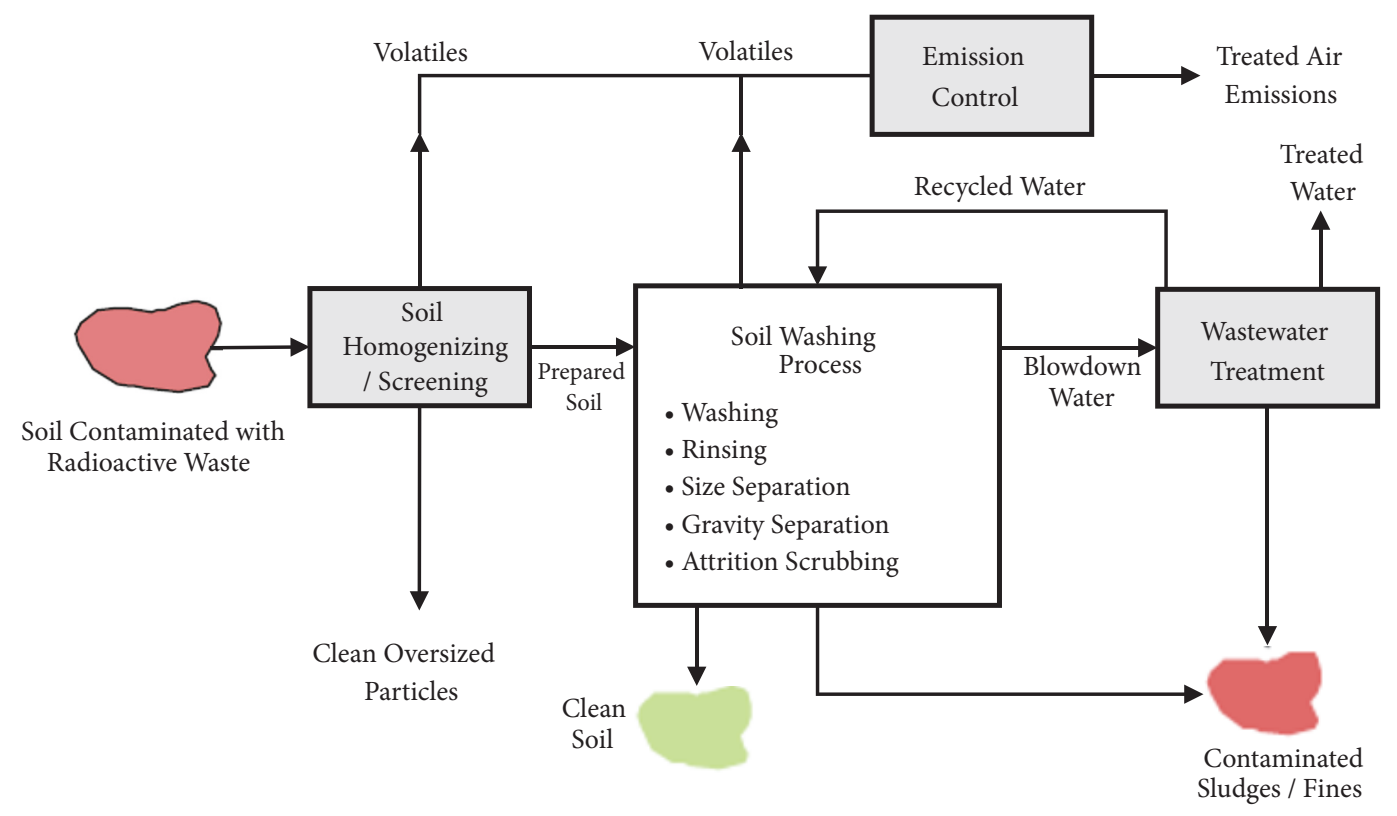

FIGURE 5: Soil washing process [5].

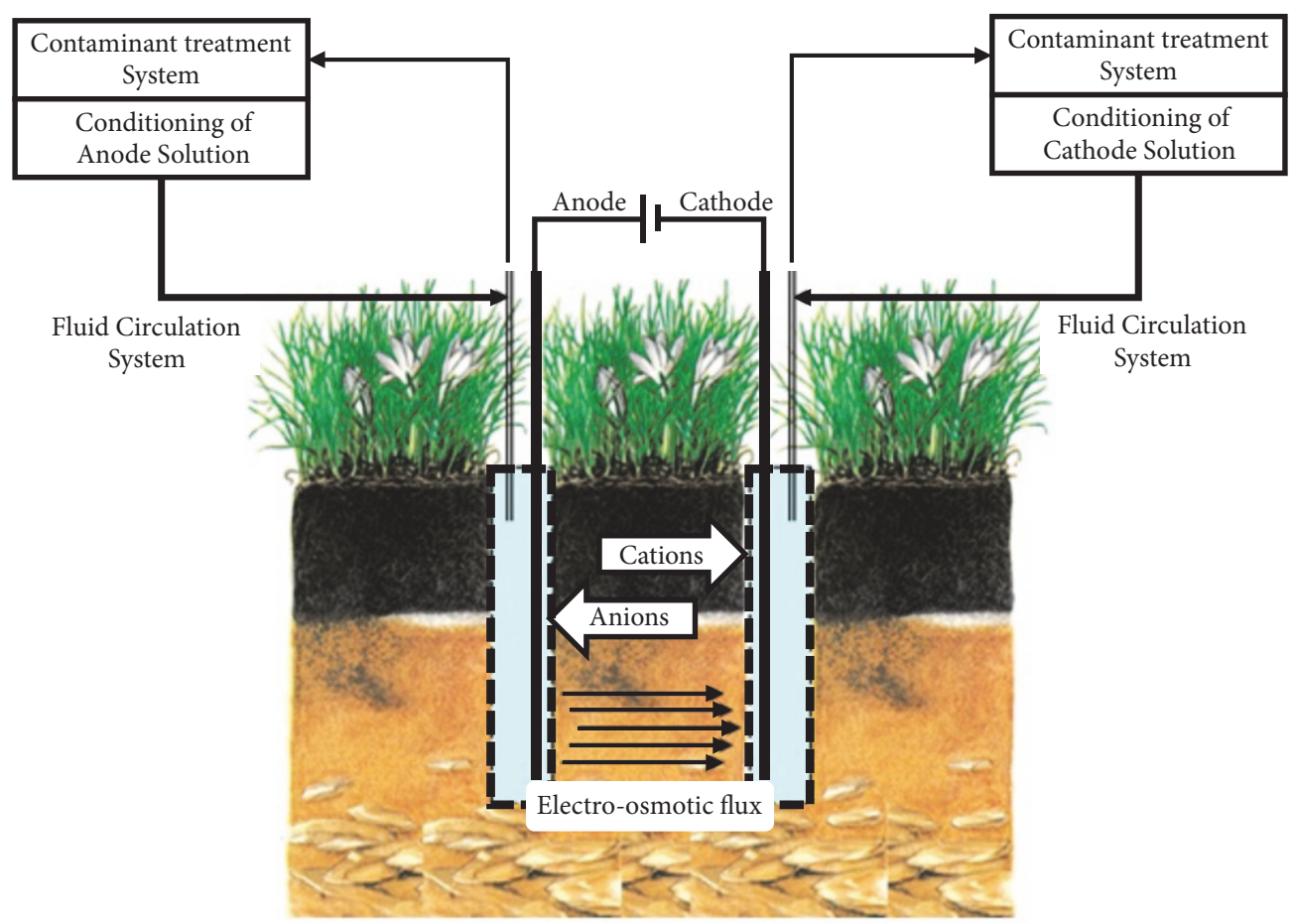

FIgURE 6: Electrokinetic remediation [6].

typical decommissioning process were modeled using the FlexSim software application. The purpose was to identify and evaluate improvements in process performance. The authors identified process rate limiting steps and other key performance parameters [13].

DES models have been used by the Sellafield Operational Research Group in number of varied nuclear waste management applications and as an overall project planning tool in decommissioning [14].
Worker radiation dose modeling has been integrated with DES models by both Los Alamos National Laboratory and the Sellafield Operational Research Group $[14,15]$.

\section{Selection of Soil Remediation Technology}

As shown in Figures 1 and 2, the soil treatment technology alternatives must first be identified and evaluated based on site-specific objectives and criteria. In general, 


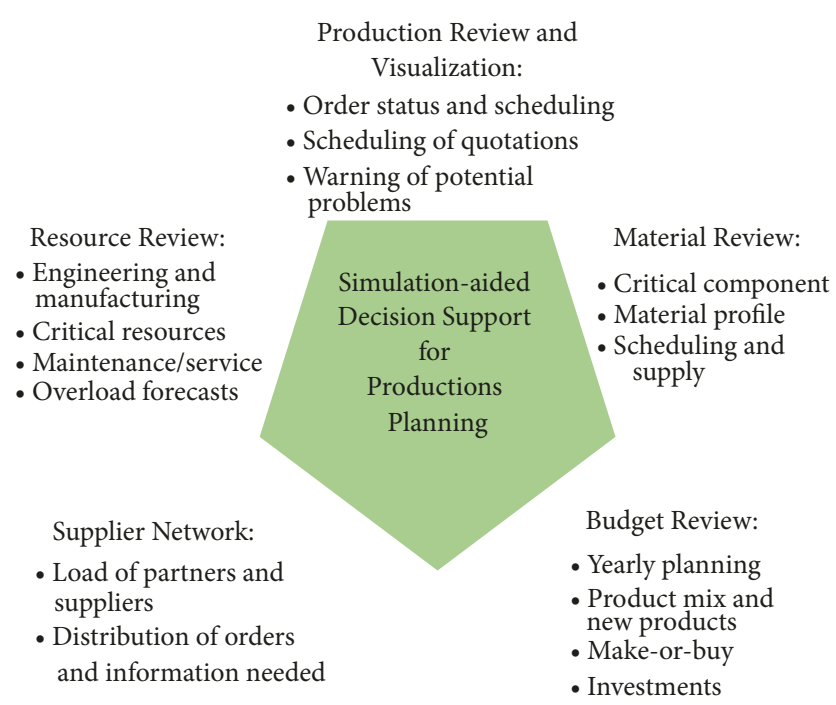

Figure 7: Potential use of simulation-aided decision support for production planning [7].

the site-specific objectives should consider (i) current or future site use, (ii) availability, appropriateness, and cost of remediation technologies, (iii) budget and timeframe, and (iv) regulatory requirements. Site investigation and characterization should be performed to determine the type and distribution of radioactive contamination. Finally, the alternative technologies should be evaluated against the technical, regulatory, and economic criteria to select the preferred alternative.

The remediation options considered in this study are solvent extraction, dry soil separation, soil washing, electrokinetic remediation, and the KAERI combined soil washing and electrokinetic separation process. The combination of dry soil separation and soil washing (hereafter referred to as soil washing) was determined to be the most appropriate technology to demonstrate the use of DES for soil remediation for Korean nuclear power plant sites.

There are several reasons for selecting a soil washing technology at the conceptual stage in developing the DES model for this study. First, the soil washing process is relatively simple and is based on the physical properties of soils. It excludes the consideration of chemical and electrical properties of contaminated soils. Second, soil washing has been used extensively in commercial applications and is easily modified to remove various radionuclides from soils. And third, the waste water from soil washing is less difficult to manage than the solvents in an extraction process. Finally, the soil washing process has a model structure similar to the KAERI ex situ electrochemical method which we think is a viable option, and a simple soil washing model could be modified to use the KAERI methodology in future applications. Soil washing is, therefore, a good technical basis for the simulation model.

\section{Soil Remediation Process}

4.1. Principles. Soil washing is a repetitive batch process that is time-consuming and has considerable uncertainty due to the lack of Korean domestic experience. It is based on the principle that contaminants are generally bound more tightly to the fine-grained particles and not to the larger course grained soil. Because of this, data for the particle size distribution of the soil at the remediation site is needed to model the soil washing process. Figure 8 shows the radioactivity concentration according to the soil particle size near a Korean nuclear facility. The figure shows the inverse relationship between particle size and radioactivity [8].

The soil washing process uses dry soil sieving in the first stage to separate the fine and course fractions prior to water washing. This reduces the contaminated soil volume prior to the washing stage potentially reducing secondary waste generation. Soil washing must be used with other treatments, such as precipitation, filtration, and/or ion exchange. Through this multistage process, the contaminated residuals (fine particles and washing solution) are treated and volume reduced for disposal. The cleaned soil that meets regulatory clearance requirements could be returned to the site and reused as backfill.

4.2. Operational Procedure. Soil washing systems normally use a six-step process [9]:

(1) Pretreatment: large size material such as rubble is removed, or optionally crushed, and scrubbed if necessary.

(2) Separation: course and fine grain soils are separated.

(3) Coarse-grained treatment: the remaining fine grain material is separated

(4) Fine-grained treatment: silt is separated from clay.

(5) Process water treatment: process water is recycled or treated for disposal.

(6) Residuals management: remediated soil can be recycled as backfill at the nuclear site. Fines and sludge are contaminated and must be managed as radioactive waste.

Soil washing processes range from relatively simple methods with several particle separation processes to complex methods including several additional processes such as magnetic or chemical treatments. However, simple soil washing processes are commonly used in the most soil washing systems and they generally remove fine fractions from the bulk soil. Simple designs have a combination of screening, classification and solids dewatering. The typical equipment used for most soil washing processes is summarized in Table 1 [2].

After the completion of soil separation according to its particle sizes using equipment listed in Table 1, additional equipment should be considered for treatment of concentrated residue and process water. In order to discharge the concentrated residue from soil washing system, the clay is subjected to dewatering process through press filter equipment. For the treatment of process water which is essential in physical separation processes, ion exchangers can be used. Water is continuously consumed during the operation, so make-up water is supplied and used process water is recycled in the system during operation [2]. 


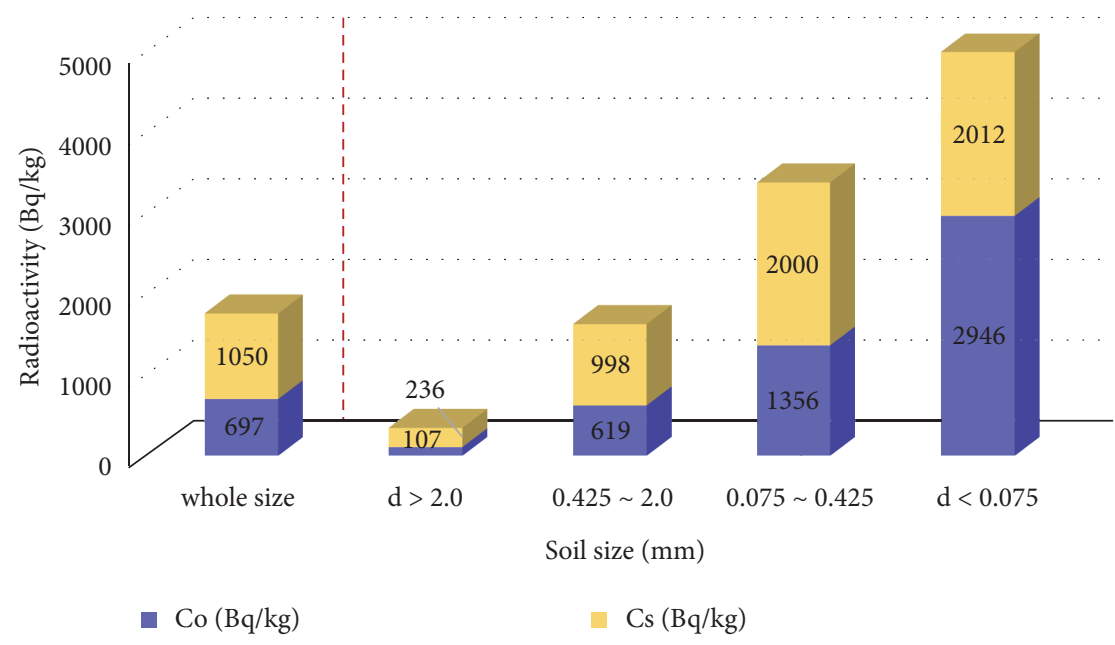

FIGURE 8: Radioactivity concentration versus particle size [8].

TABLE 1: Typical soil washing equipment [2].

\begin{tabular}{lc}
\hline Exploitable Soil & Process Equipment \\
\hline Size & Vibratory Screens (sieves) \\
& Sieve Bends \\
& Trommel (rotary) Screens \\
\hline Hydraulic Size (settling Velocity) & Classifiers \\
& Hydrosizers \\
& Hydrocyclones \\
Specific Gravity & Jigs \\
& Sluices \\
& Dense Media Separators \\
Surface Chemistry & Spirals \\
\hline
\end{tabular}

\section{FlexSim Simulation Model}

5.1. Process Model. The soil washing process has two main objectives. These are to reduce the volume of waste through separation by particle size and to properly manage the concentrated residues. The process in the diagram (Figure 9) is a relatively simple abstraction that contains only elements that are essential to meet the two main objectives.

The first step in developing the simulation model is to visualize the target system through schematization. The simplified soil washing system is expressed in Figure 9 as a process flow block diagram.

Each block represents the equipment used in the soil washing process and the work being performed in each stage. Black and blue arrows, respectively, indicate the flow of soils and wash water. The black arrow implies process logic such as the distinction and separation of the soil flow according to particle sizes. Soil screening and an attrition scrubber are used for the pretreatment phase; two sand screws, a cyclone, and a filter press, are used for the separation phase. It is assumed that the treatments of coarse- and fine-grained soils, such as rinsing and dewatering, are included in each of the blocks for simplification of the model. Wash water is injected to each block for separation and circulated through a water treatment system. Additional water must be injected to compensate for water lost with the cleaned soil. Finally, the treated sludge/residue must be managed as radioactive waste.

5.2. FlexSim Model Development Approach. A simulation model of the soil washing process was developed using FlexSim, a discrete event simulation modeling application that is widely used in industries such as material handling, manufacturing, logistics, transportation, and mining [16]. In this study, a simple process model representing a soil remediation plant was developed to demonstrate the use of simulation for planning a soil remediation process.

To be effective, simulation development should use a systematic approach. In this study, development of the simulation used the following approach [7]:

(i) Project definition

(ii) Process mapping (static diagram)

(iii) Simulation model (dynamic)

(iv) Verification

(v) Simulation of cases for study

(vi) Findings, conclusions, and recommendations

5.3. FlexSim Model Assumptions. The simulation model has several simplifying assumptions as follows:

(i) Fifteen tons of soil can be treated per a batch. The system has 240 tons/day processing capacity under 8hour operation a day.

(ii) There is no need for warming-up between the idle state and steady state. In the simulation, the system equipment enters the steady state immediately with the beginning of operation.

(iii) Due to lack of detailed commercial data except for general information about overseas experience, 


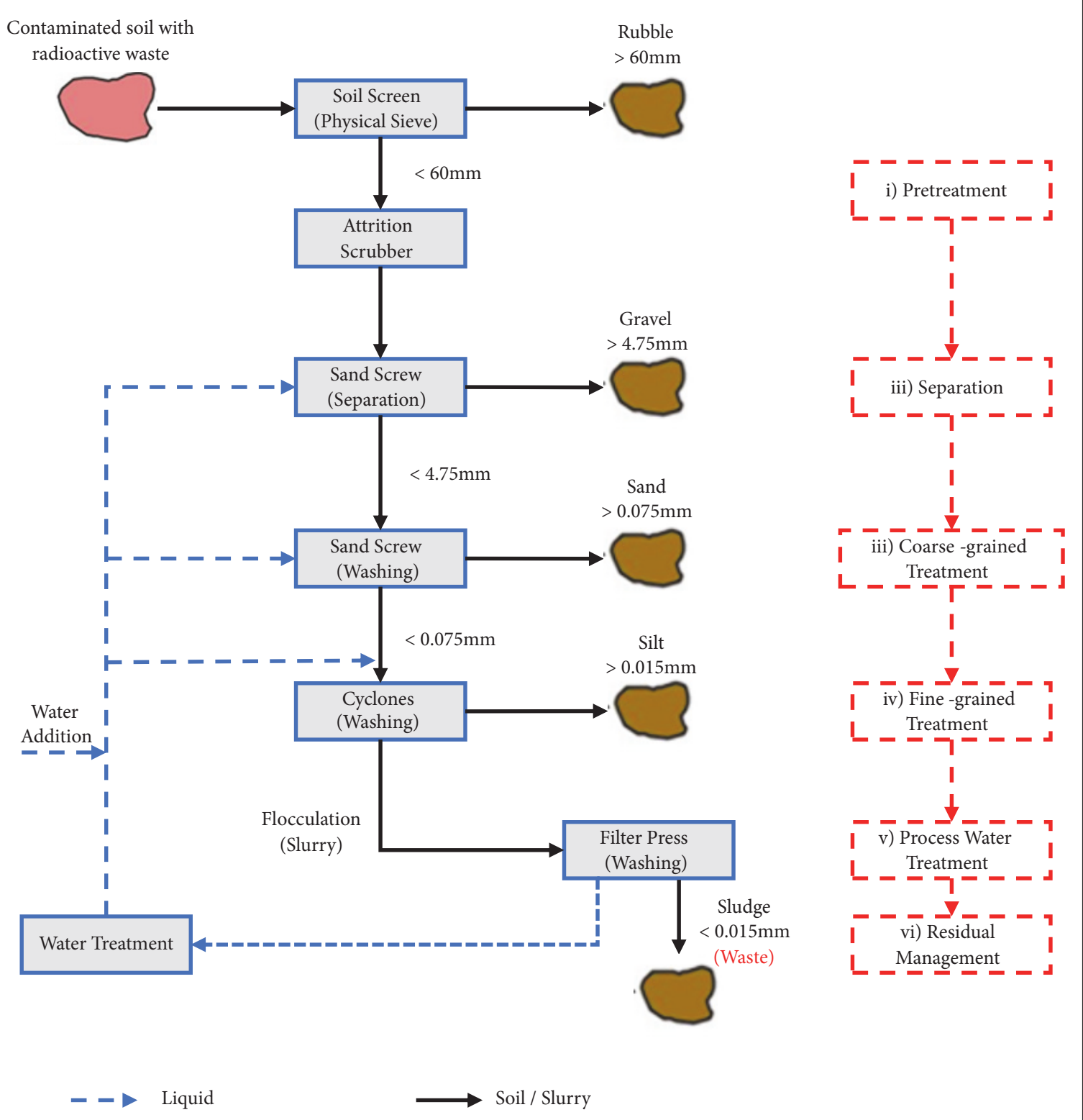

FIGURE 9: Soil washing process model [9].

assumptions are applied to the process rate and capacity for system components and the overall process.

(iv) The system has enough capacity to operate without bottlenecks.

(v) There are no unplanned shutdowns due equipment failure or maintenance activities.

In the development of the simulation model, among various resources and functions of FlexSim, four basic objects were used to visualize the soil remediation process. Those objects are sources, queues, processors, and sinks and are defined as follows [16].

Source. The source creates flow items and releases them to a downstream object. The simulation user can control the rate at various nodes at which the source creates flow items so that they arrive on a fixed schedule, a regular continuous rate, or a random statistical distribution. In the reference model, a fixed (scheduled) arrival scenario simulates the batch process.

Queue. The queue stores flow items until a downstream object is ready to take them. By default, the queue releases flow items on a first-in-first-out basis, but other options are available.

Processor. Processors simulate flow items being processed at a station. Processors simulate a time delay, beginning with a setup time followed by the process time. The user can also require the processor to use an operator during the setup and/or process time. The user can also set processors to handle more than one flow item at a time. In the reference model, constant process times are used. 


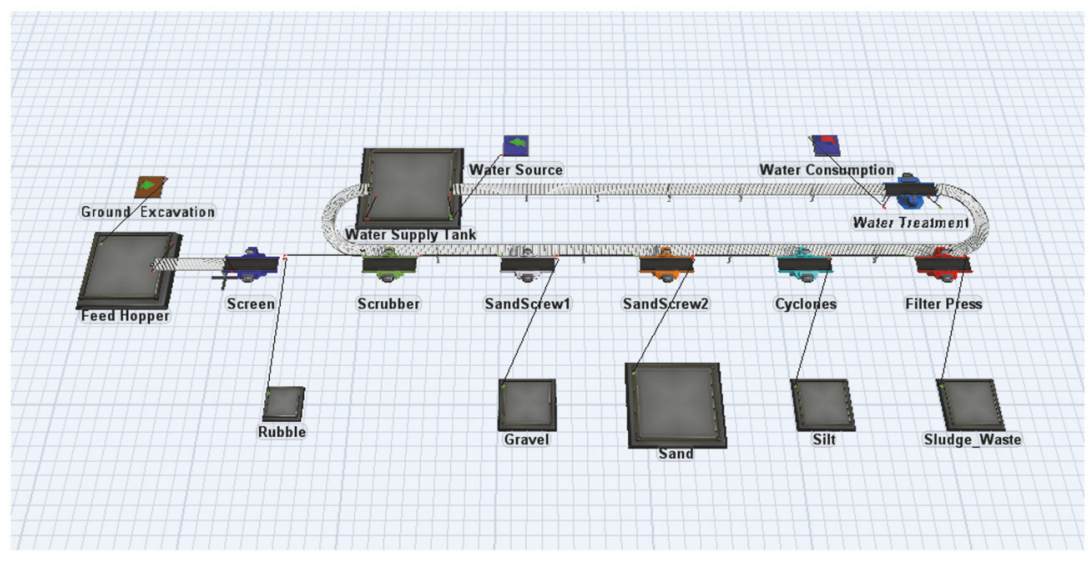

FIGURE 10: Logistical simulation of soil washing process.

Sink. The sink removes flow items from a simulation model when the items passed the final process.

In the simulation model the key elements control the movement of contaminated media (soil and wash water) as operational characteristics of the system. The target media are generated from the sources then transferred and separated according to the intended logic in the simulation.

5.4. System Layout. The soil washing process is visualized in the simulation model using FlexSim software based on the process flow block diagram as shown in Figure 10. The simulation was constructed by creating objects and connecting them according to the flow of soil within the soil washing system.

Figure 10 shows a screenshot of the soil washing simulation model from the 3D output of the FlexSim model. The excavated soil from the contaminated site is fed to the feed hopper on the left side of the figure, and the soil that has been cleaned through dry separation is sent to each queue according to the grain size, i.e., rubble, gravel, sand, silt, and sludge/clay. While the separation and treatment of soil are proceeding, wash water is circulating within the water circulation system located in the upper part of the figure. Some of the wash water is consumed, because water is contained in cleaned (discharged) soil. To compensate for water loss, make-up water is injected to the water supply tank.

5.5. Simulation Logic. Once the physical structure of the simulation model is set, the next step is to create the logic that determines how the soil and water flows into and through the simulation model. In the soil washing process, each equipment element is designed to separate soils according to their particle size.

5.6. Flow of Soils. In the simulation model, the processor objects are used to represent the components in the soil washing process. First, variables are designated as the soil "item" according to the attributes of each unit soil that will enter the system. Then each processor located downstream classifies and cleans the soil based on the input variables. For example, "Sandscrew2" (Figure 10) classifies sand particles with a value " 3 " for the "item.type" variable and sends them to the "queue" and sends soil particles other than sands to the next element, "processor: cyclones".

The particle size distribution of the soil excavated at the site must be determined. Preliminary soil particle size distribution data obtained from soil samples around the perimeter of the Kori site by KAERI was used to obtain a more realistic as it is the best available preliminary data [2]. Table 2 lists the raw soil data, the soil excavation/cleanup rates, and the derived input data.

The input data in Table 2 were derived from the raw data through simple processing. The input data were developed by averaging the numerical values for particle size distribution obtained from 10 samples around the Kori NPP site. A value of $1 \%$ for the rubble $(>60 \mathrm{~mm})$ was assumed. Because the main concern in the washing process is silt and clay, this assumption does not impact the results. The composition of the silt and clay in the input data column was obtained from 10 samples by estimating the values from the standard soil texture diagram [17]. Once the soil particle size distribution is input, the "source" object generates the soil "items" probabilistically according to the distribution of the input and provides the soil particles to the system.

The excavation rate to feed soil to the system and the cleanup rate to remediate the soil were also assumed. On a commercial scale, there were large nonnuclear projects with a throughput of 20-100 tons/hour [5]. In this study, the system capacity was assumed to be 240 tons/day (30 tons/hour for 8 hours a day). The faster processing speed as compared to excavation speed is an intentional assumption to avoid bottlenecks in the system.

5.7. Flow of Wash Water. In the model, the wash water system was schematized but not integrated with the soil treatment system. This was simplifying assumption and for their integration additional "processors" for combining and separating soil and water will be needed at junction points such as scrubbers, screws, cyclones, and filter presses.

As soils are cleaned by soil washing, the soil moisture content increases. The moisture content of the soil is the ratio of the weight of the water in the soil. In this simulation, 
TABLE 2: Soil input data for the model [2].

\begin{tabular}{|c|c|c|c|c|}
\hline \multirow{2}{*}{ Parameters } & \multicolumn{2}{|c|}{ Raw Data } & \multicolumn{2}{|c|}{ Input Data } \\
\hline & Type & Value & Type & Value \\
\hline Excavation Rate & - & - & - & 30.0 tons $/ \mathrm{hr}$ \\
\hline \multirow{5}{*}{ Particle Size of Soil } & \multirow{2}{*}{ Gravel $(>4.75 \mathrm{~mm})$} & \multirow{2}{*}{$6.3 \%$} & Rubble (>60mm) & $1.0 \%$ \\
\hline & & & Gravel $(60 \sim 4.75 \mathrm{~mm})$ & $5.3 \%$ \\
\hline & Sand $(4.75 \sim 0.075 \mathrm{~mm})$ & $83.4 \%$ & Sand $(4.75 \sim 0.075 \mathrm{~mm})$ & $83.4 \%$ \\
\hline & \multirow{2}{*}{ Silt \& Clay $(<0.075 \mathrm{~mm})$} & \multirow{2}{*}{$10.3 \%$} & Silt $(0.075 \sim 0.015 \mathrm{~mm})$ & $3.9 \%$ \\
\hline & & & Clay $(<0.015 \mathrm{~mm})$ & $6.4 \%$ \\
\hline Cleanup Process Rate & - & - & - & 36.0 tons $/ \mathrm{hr}$ \\
\hline
\end{tabular}

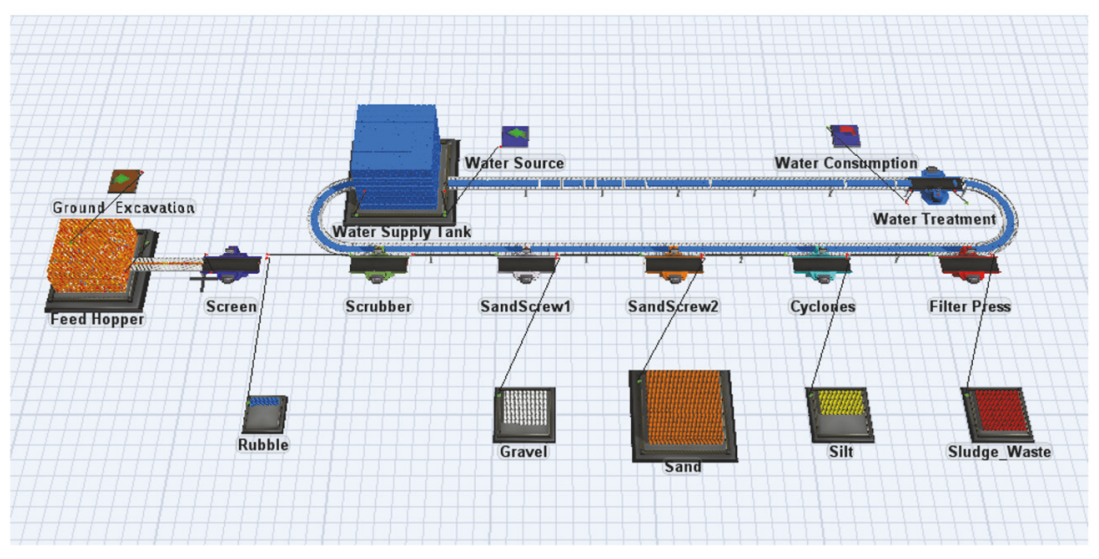

FIGURE 11: Logistical simulation model operation.

increase of soil moisture content is assumed as $10 \%$. That means that 3 tons of water is consumed per batch and 3 tons of make-up water is supplied to the water supply tank. Recycling of wash from dewatering during the process could be included in future revisions of the model.

5.8. Verification. Verification was performed to assure that the simulation was working as intended.

In this study, the simulation model was verified by checking the output of the simulation. In the simulation, we can observe the visualized soil washing process and the waste throughput. Once the simulation starts, 30 tons of soil per hour is supplied to the feed hopper and the soil washing system operates at 36 tons per hour. Due to difference between the soil excavation rate and process rate, this system can be maintained as a 1-hour batch process.

The object for comparison is the particle size distribution that was input into the "source" object. Figure 11 shows a screenshot during operation of simulation. In the feed hopper on the left of the figure, soil particles in different colors are mixed randomly according to the probabilistic input data for soil particle size distribution. They are separated and treated by "processors" according to their values in "item.type" variable. As a result of simulation, separated and cleaned soils are stacked in the "queues".

Table 3 shows the process results after 2 hours of operation. The results show that the process output is approximately equal to the input meaning the simulation is operating correctly according to the intended logic. The model generates detailed processing data as a numerical table or graphical chart as shown in Figure 12.

The system is shown to be operating in a batch process as seen in Figure 12 which shows that the soil in the feed hopper accumulates and is emptied repeatedly. The State Pie diagram in Figure 12 shows utilization of the system equipment elements.

\section{Discussion}

The purpose of this report is to suggest that the decommissioning and site remediation projects can be optimized and improved by applying simulations to the soil remediation planning. In this section we discuss the application of simulation to the soil remediation planning.

In a soil washing system batch or continuous operation has advantages and disadvantages. In this study, the simulation model used the batch process condition by controlling the excavation rate and cleanup rate. The difference between the batch and the continuous is not significant in system operation. However, due to radioactive waste management regulatory requirements may be necessary to process the soils in batches based on site characterization and waste classification. Batch operation can avoid the regulatory issues related to blending radioactive wastes with different classifications.

Bottlenecks are a major concern in terms of efficient operation of the system. Looking at the "State Pie" in Figure 12, cyclones and filter presses at the downstream are relatively free because the idle state is dominant, while 

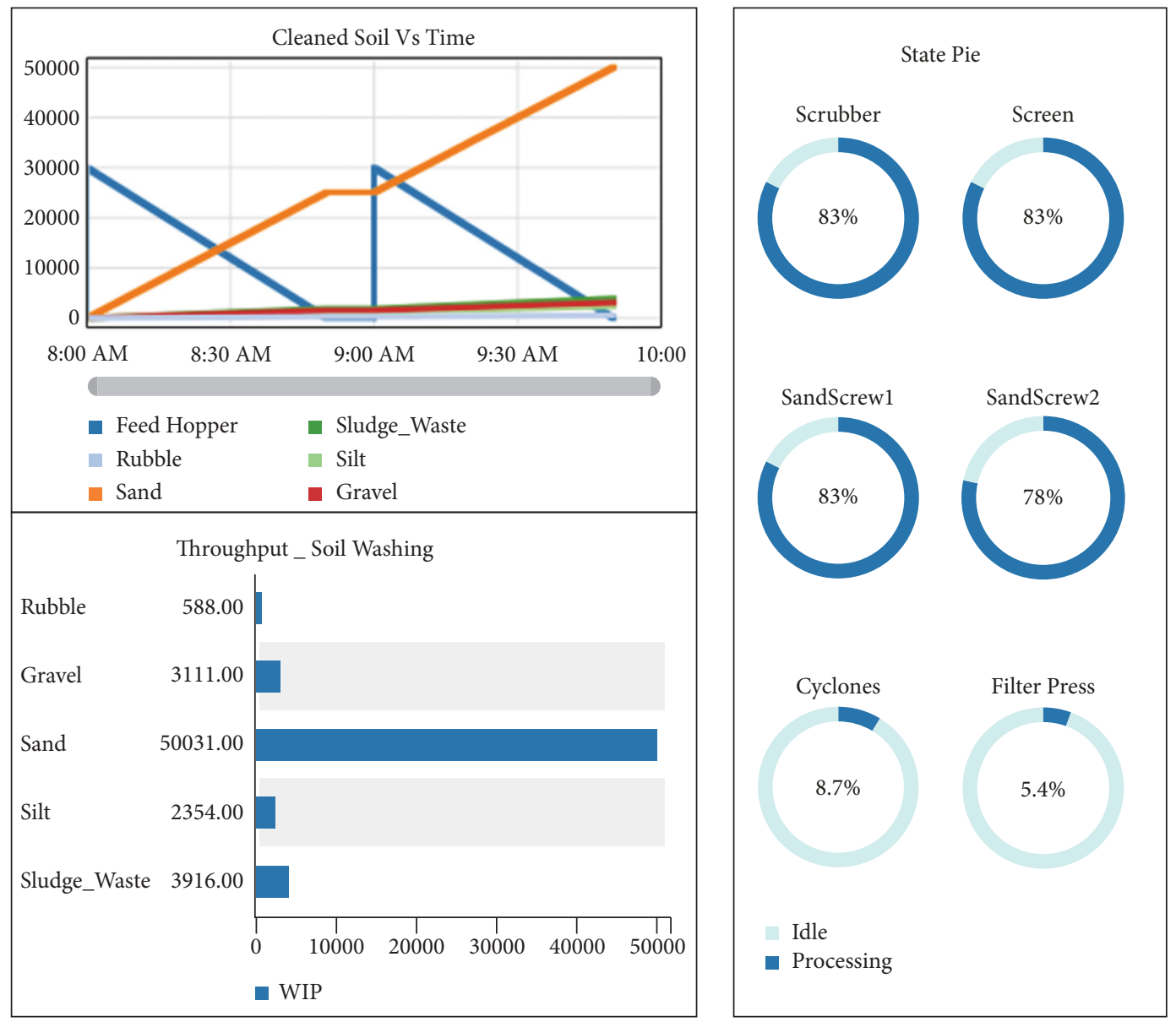

FIGURE 12: Graphical report for soil washing (at 2 hours).

TABLE 3: Simulation results at 2 hours of operation.

\begin{tabular}{lcccccc}
\hline Queue & Rubble & Gravel & Sand & Silt & Sludge & Total \\
\hline Processed Soil (ton) & 0.588 & 3.111 & 50.031 & 2.354 & 3.916 & 60.000 \\
\hline Ratio (\%) & $0.98 \%$ & $5.19 \%$ & $83.39 \%$ & $3.92 \%$ & $6.53 \%$ & $100.00 \%$ \\
\hline Input Data (\%) & $1.0 \%$ & $5.3 \%$ & $83.4 \%$ & $3.9 \%$ & $6.4 \%$ & $100.00 \%$ \\
\hline Error (\%) & $-2.00 \%$ & $-2.08 \%$ & $-0.01 \%$ & $0.51 \%$ & $2.03 \%$ & - \\
\hline
\end{tabular}

four upstream devices have very high utilization. This means that the upstream devices are relatively overloaded and the downstream devices are relatively overdesigned.

One way to prevent bottlenecks is to increase the device's capacity, but in terms of system maintenance and reliability, it may be better to distribute the flow into parallel devices rather than a single larger device. This preliminary simulation model does not include the failure of the equipment or the downtime due to maintenance; however a more detailed simulation model will be able to evaluate system component failure and scheduled downtime.

Figure 13 depicts a soil remediation and waste management system. In this study we have modeled the soil washing process (Module 2). Module 1 represents soil excavation module in which excavated soil from the contaminated site is transferred to a soil treatment system to clean the soil. In Module 3, the waste management system is shown downstream from the soil remediation process. For future development of this model, we should develop Module 1 and Module 3.

Process optimization requires making decisions in a comprehensive way, including cost, schedule, and resources such as personnel and equipment. Figure 7 shows the potential for using simulation in the planning phase [13]. We can view the soil remediation system as a production system with nuclear waste as the product. As we further develop our soil remediation simulation model, we should include more detail that will allow a holistic evaluation of the waste production system.

\section{Conclusions}

In this study, we investigated the applicability of discrete event simulation for a soil washing process for planning 


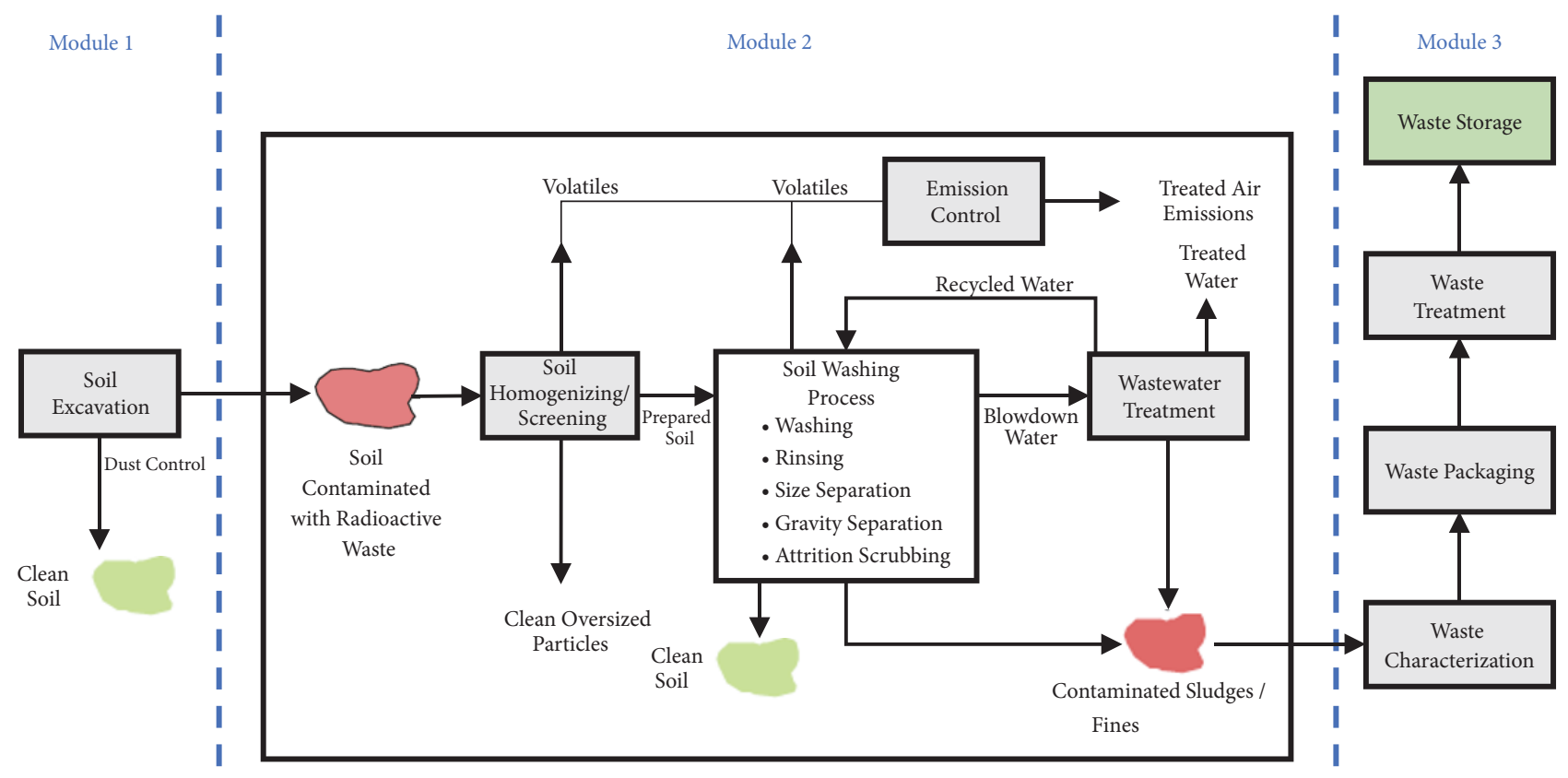

FIGURE 13: Expanded soil remediation process model.

site remediation. It was shown that a batch process such as soil washing can be effectively modeled as a discrete event process. Based on the literature review, efficient allocation of resources and efficient waste management including volume reduction can be achieved by use of the model for planning the soil remediation process.

Furthermore, we suggest extending the scope of the simulation model. The scope could extend beyond soil remediation to include the entire site remediation process from site excavation to waste management. This suggests that the simulation model can be used as a tool for decision analysis and planning of nuclear power plant decommissioning and remediation projects.

In future work, a conceptual site model (CSM) as suggested by NEA (Figure 2) should be developed to define the site-specific problems to be solved by remediation. Site characterization data should be incorporated in the CSM when such data becomes available for Kori 1. At that time, a more detailed simulation model can be developed to include site soil properties, radionuclides of interest, and a specific soil washing process. More broadly, site remediation starts with a site investigation and development of the site conceptual model and shows possibilities for the integration of excavation, soil treatment, waste characterization, packaging, storage, and worker dose exposure assessment.

\section{Data Availability}

The data used to support the findings of this study are included within the article.

\section{Conflicts of Interest}

The authors declare that they have no conflicts of interest.

\section{Acknowledgments}

This research was supported by the Nuclear Safety Research Program through the Korea Foundation of Nuclear Safety (KOFONS) and granted financial resource from the Nuclear Safety and Security Commission (NSSC), Republic of Korea (no. 1605008).

\section{References}

[1] International Atomic Energy Agency, Integrated Approach to Planning the Remediation of Sites Undergoing Decommissioning, IAEA, Vienna, Austria, 2009.

[2] B. Seo, I. Kim, J. Nam et al., Development of Decommissioning, Decontamination and Remediation Technology for Nuclear Facilities Development of Site Remediation Technology for Decommissioning and Contaminated Site, Korea Atomic Energy Research Institute, KAERI/RR-4233/2016, 2017.

[3] Nuclear Energy Agency, Nuclear Site Remediation and Restoration during Decommissioning of Nuclear Installations, OECD Publishing, NEA No. 7192, 2014.

[4] Electric Power Research Institute, Power Reactor Decommissioning Experience, Palo Alto, Calif, USA, 2008.

[5] U.S. Environmental Protection Agency, Technology Reference Guide for Radioactively Contaminated Media, 2007.

[6] C. Cameselle, S. Gouveia, D. E. Akretche, and B. Belhadj, "Advances in electrokinetic remediation for the removal of organic contaminants in soils," Organic Pollutants - Monitoring, Risk and Treatment, 2013.

[7] J. Heilala, J. Montonen, P. Jarvinen et al., "Developing simulation-based Decision Support Systems for customer-driven manufacturing operation planning," in Proceedings of the 2010 Winter Simulation Conference - (WSC 2010), pp. 3363-3375, Baltimore, MD, USA, 2010. 
[8] G.-N. Kim, S.-S. Lee, D.-B. Shon, K.-W. Lee, and U.-S. Chung, "Development of pilot-scale electrokinetic remediation technology to remove $60 \mathrm{Co}$ and $137 \mathrm{Cs}$ from soil," Journal of Industrial and Engineering Chemistry, vol. 16, no. 6, pp. 986-991, 2010.

[9] U.S. Environmental Protection Agency, Soil Washing/Soil Flushing Volume 3, U.S. Environmental Protection Agency, EPA 542B-93-012, 1993.

[10] G.-N. Kim, S.-S. Kim, and J.-W. Choi, "Removal of 137cs from contaminated soil using pilot electrokinetic decontamination equipment," International Journal of Environmental and Agriculture Research, vol. 3, no. 1, p. 7, 2017.

[11] Electrokinetic Separation, The Federal Remediation Technology Roundtable (FRTR), 2018, https://frtr.gov/matrix2/section4/ 4-4.html.

[12] C. W. Alexander, "Discrete event simulation for batch processing," in Proceedings of the 2006 Winter Simulation Conference, pp. 1929-1934, Monterey, Calif, USA, 2006.

[13] G. Dottavio, M. F. Andrade, F. Renard, and V. Cheutet, "Logistical optimization of nuclear waste flows during decommissioning," International Journal of Environmental Ecological and Engineering, vol. 10, no. 10, p. 6, 2016.

[14] R. Thompson and S. McCann, "The application of simulation modeling in nuclear decommissioning," in Proceedings of the WM2010 Conference, p. 7, AZ, Tucson, USA, 2010.

[15] G. H. Tompkins, D. E. Kornreich, R. Y. Parker, A. C. Koehler, J. M. Gonzales-Lujan, and R. J. Burnside, "Dynamic radiation dose visualization in discrete-event nuclear facility simulation models," in Proceedings of the 2004 Winter Simulation Conference, pp. 472-478, Washington, DC, USA, December 2004.

[16] FlexSim User Manual, Flexsim Software Products, Inc., 2017.

[17] "Soil Texture Calculator - NRCS Soils," https://www.nrcs.usda .gov/wps/portal/nrcs/detail/soils/survey/?cid=nrcs142p2_054167. 

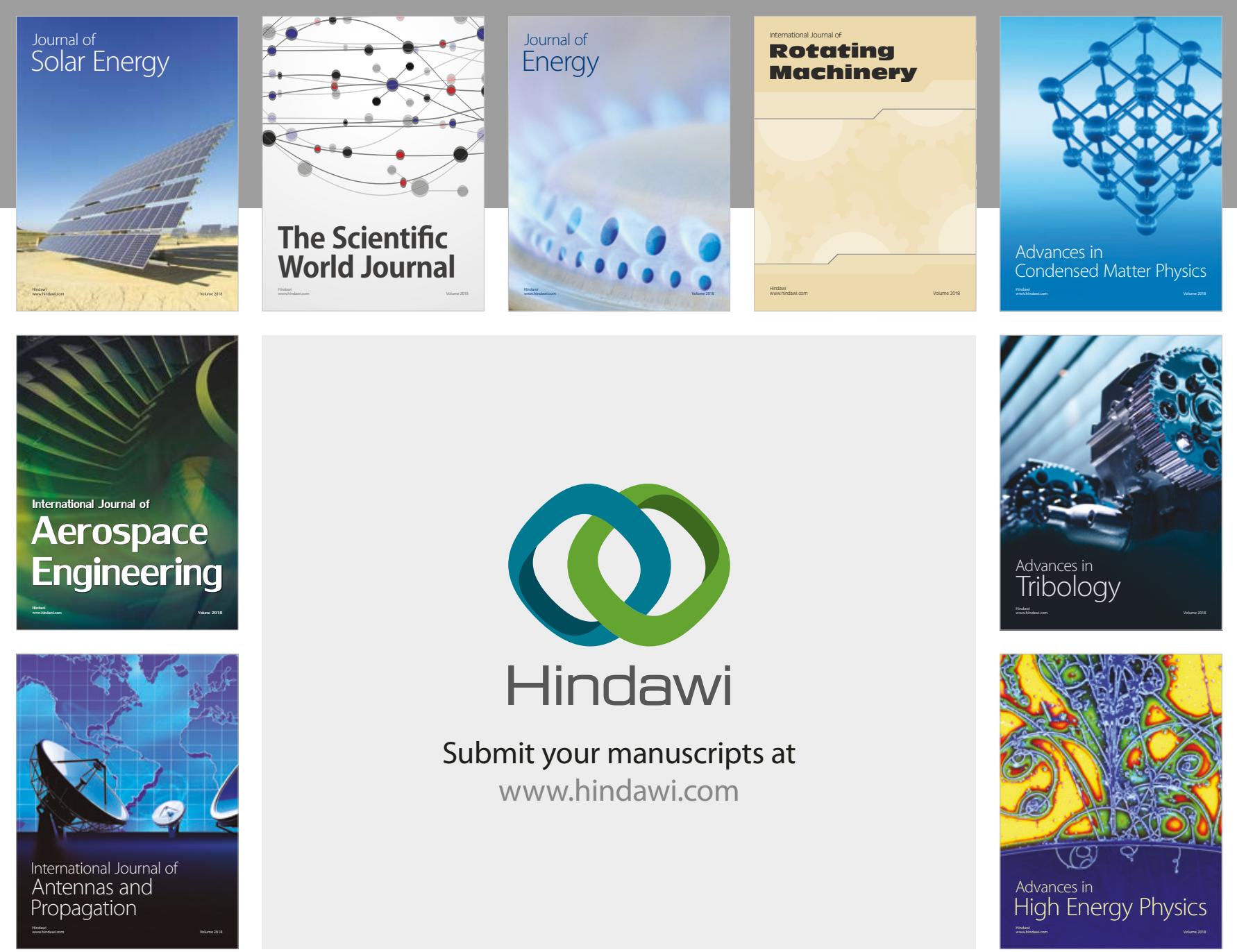

Submit your manuscripts at

www.hindawi.com
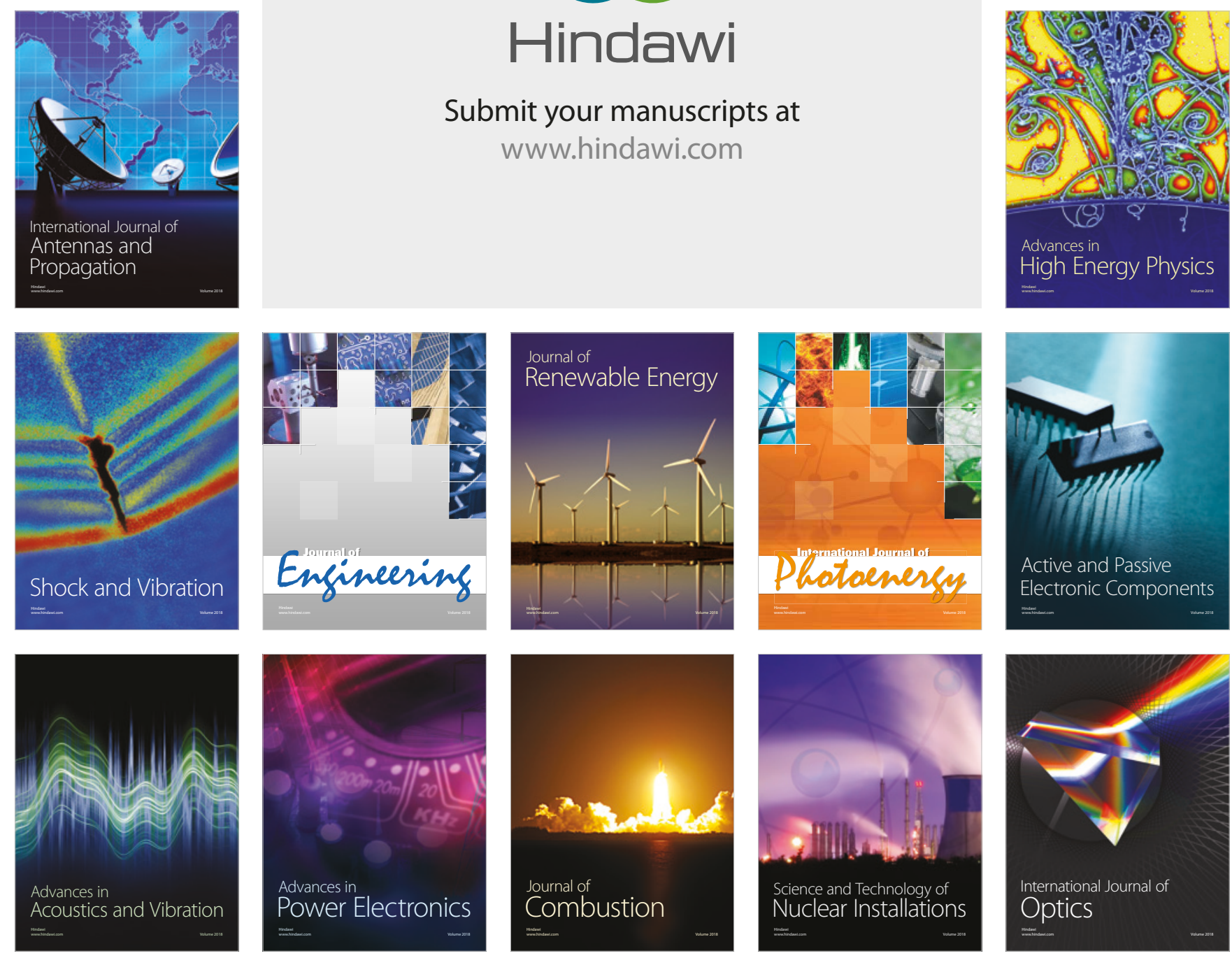